\title{
Avaliação da microestrutura e das propriedades magnéticas de ferritas Ni-Zn dopadas com cobre
}

\author{
(Evaluation of microstructure and magnetic properties \\ of Ni-Zn ferrite doped with copper)
}

\author{
R. A. Torquato ${ }^{I}$, F. A. Portela ${ }^{2}$ L. Gama ${ }^{I}$, D. R. Cornejo ${ }^{3}$, S. M. Rezende , R. H. G. A. Kiminami ${ }^{5}$ A. C. F. M. Costa ${ }^{1}$ \\ ${ }^{1}$ Laboratório de Síntese de Materiais Cerâmicos - LabSMac, Departamento de Engenharia de Materiais, Universidade \\ Federal de Campina Grande, Av. Aprígio Veloso 882, Bodocongó, Campina Grande, PB 58109-970 \\ ${ }^{2}$ Departamento de Química, Universidade Federal da Paraíba, J. Pessoa, PB 58000-000 \\ ${ }^{3}$ Instituto de Física, Universidade de S. Paulo, S. Paulo, SP 05508-900 \\ ${ }^{4}$ Departamento de Física, Universidade Federal de Pernambuco, Recife, PE 50670-901 \\ ${ }^{5}$ Departamento de Engenharia de Materiais, Universidade Federal de S. Carlos \\ Rod. Washington Luiz, km 235, C.P. 676, S. Carlos, SP 13565-905
}

\begin{abstract}
Resumo
A microestrutura e as propriedades magnéticas das ferritas $\mathrm{Ni}_{0,5-\mathrm{x}} \mathrm{Cu}_{\mathrm{x}} \mathrm{Zn}_{0,5} \mathrm{Fe}_{2} \mathrm{O}_{4}$ com $\mathrm{x}=0,1 / 0,2 / 0,3 / 0,4 \mathrm{~mol}$ de cobre, preparadas a partir de pós obtidos por reação de combustão com tamanho de partícula na faixa de $23 \mathrm{a} 29 \mathrm{~nm}$, foram avaliadas. Os pós obtidos foram prensados uniaxialmente e sinterizadas a $1000^{\circ} \mathrm{C} / 2 \mathrm{~h}$. As amostras foram caracterizadas por medidas de densidade e porosidade aparente, difração de raios X, microscopia eletrônica de varredura e medidas magnéticas M-H. Os difratogramas de raios $\mathrm{X}$ mostram a presença da fase majoritária cristalina do espinélio inverso em todos os sistemas, e traços da fase secundária $\mathrm{Fe}_{2} \mathrm{O}_{3}$ nas amostras com $\mathrm{x}=0,1$ e 0,4 . O aumento do teor de cobre causou um pequeno aumento no tamanho médio de grão $(0,65$ para $0,68 \mu \mathrm{m})$, uma redução da porosidade aparente $(33,7 \%$ para $6,6 \%)$ e redução da magnetização de saturação de 69 para 54 emu/g.

Palavras-chave: ferrita Ni-Zn, propriedades magnéticas, microestrutura.
\end{abstract}

\begin{abstract}
An evaluation was made of the microstructure and magnetic properties of $\mathrm{Ni}_{0.5-x} \mathrm{Cu}_{x} \mathrm{Zn}_{0,5} \mathrm{Fe}_{2} \mathrm{O}_{4}$ ferrites with $\mathrm{x}=0.1,0.2$, 0.3 and 0.4 prepared by combustion reaction with particle sizes 23 to $29 \mathrm{~nm}$. The resulting powders were uniaxially compacted at $385 \mathrm{MPa}$ into pellets and sintered at $1000^{\circ} \mathrm{C} / 2 \mathrm{~h}$. The samples were characterized by apparent density and porosity measurements, $X$-ray diffraction, scanning electron microscopy and $M-H$ magnetic measurements. The $X$-ray diffraction revealed crystalline inverse spinel as the major phase in all the specimens and a small amount of hematite $\left(\alpha-\mathrm{Fe}_{2} \mathrm{O}_{3}\right)$ as the secondary phase in the $x=0.1$ and 0.4 samples. An increase in the amount of copper caused a slight increase in grain size (0.65 to $0.68 \mu \mathrm{m})$, a decrease in the apparent porosity (33.7\% to $6.6 \%)$ and a reduction in saturation magnetization from 69 to $54 \mathrm{emu} / \mathrm{g}$.
\end{abstract}

Keywords: Ni-Zn ferrite, magnetic properties, microstructure.

\section{INTRODUÇÃO}

As ferritas Ni-Zn são materiais cerâmicos ferrimagnéticos, também conhecidos como materiais magnéticos moles ou macios (soft), cuja estrutura cristalina é semelhante à do mineral espinélio. O desempenho das ferritas Ni-Zn não é determinado apenas pelos altos valores de resistividade e permeabilidade magnética inicial, mas também por outras características, tais como baixos valores de perdas por histerese e correntes parasitas (Foucault), altas densidades de fluxo de saturação, baixos campos coercitivos e alta permeabilidade magnética final em altas freqüências (10$500 \mathrm{MHz}$ ), dureza mecânica, estabilidade química e custo relativamente baixo [1-5].

Atualmente, materiais com propriedades ferrimagnéticas vêm desempenhando papel de grande importância no campo científico devido às suas inúmeras aplicações tecnológicas, tais como transformadores de potência e de pulso, transformadores para distribuição de energia, transformadores de banda larga e baixa potência, geradores, fontes de potência, indutores e transdutores, filtros de freqüência variável e supressores de ruído [1-2]. A 
aplicação na eletrônica de potência de materiais cristalinos magneticamente moles como ferritas de Ni-Zn, vem se incrementando constantemente. Em particular, o aumento do mercado comercial para fontes de potência exige a produção de núcleos de ferritas de alta performance para operar em freqüências cada vez maiores e com menos perdas. Isto tem levado a um forte incremento na pesquisa básica e aplicada de propriedades magnéticas de ferritas Ni-Zn com diferentes dopantes, para aplicações em altas freqüências, com o intuito de miniaturizar componentes elétricos e eletrônicos. Com o crescimento das pesquisas em Nanociências e Nanotecnologia e a decorrente necessidade de componentes elétricos e eletrônicos cada vez menores, tem se verificado um renovado impulso na busca de novos materiais magnéticos e nos estudos tendentes a otimizar os materiais conhecidos [6].

A influência do cobre sobre algumas composições de ferritas tem sido bastante estudada por vários pesquisadores, ao longo das últimas décadas, com o intuito de melhorar algumas propriedades e características destes materiais. Por outro lado, as ferritas dopadas com cobre têm despertado interesse da indústria eletrônica devido às boas propriedades elétricas e magnéticas em dispositivos que atuam em altas freqüências [7]. Um dos efeitos mais marcantes da dopagem com cobre em sistemas de estrutura espinélio é a distorção tetragonal que ocorre no parâmetro de rede da célula cúbica original do espinélio, ou seja, a célula unitária do espinélio, pode sofrer um discreto alongamento em uma das arestas, quando da inclusão de certa quantidade de cobre. Este alongamento muda a simetria do sistema que passa de espinélio cúbico para tetragonal. E isto ocorre em decorrência do chamado efeito Jahn-Teller [8,9].

Em pesquisa sobre a influência do cobre sobre as propriedades elétricas e magnéticas das ferritas $\mathrm{Mg}_{0.5-\mathrm{x}} \mathrm{Cu}_{\mathrm{x}} \mathrm{Zn}_{0.5} \mathrm{Fe}_{2} \mathrm{O}_{4}+0,5 \mathrm{MgO}$ com $\mathrm{x}=0,0 / 0,2 / 0,3 / 0,4 / 0,5 \mathrm{~mol}$, sintetizado pelo método convencional de mistura de óxidos, os autores confirmaram o papel importante do cobre no processo de densificação e sinterização de ferritas. Foi verificado que a densidade final aumentou com o aumento do teor de cobre de $\mathrm{x}=0,1$ a $\mathrm{x}=0,3$. Acima deste valor foi observada uma diminuição na densificação, atribuída ao aumento da porosidade intragranular, resultando em um descontínuo crescimento do grão. A magnetização de saturação aumentou quase linearmente com o aumento do teor de $\mathrm{Cu}^{2+}$, visto o cobre possui resultante magnética 1 $\mu_{\mathrm{B}}$, enquanto o $\mathrm{Mg}^{2+}$ não apresenta nenhuma resultante de momento magnético [10]. O efeito da substituição do níquel por cobre sobre as propriedades elétricas e magnéticas em ferritas $\mathrm{Ni}-\mathrm{Zn}\left(\mathrm{Ni}_{0,8-\mathrm{x}} \mathrm{Cu}_{\mathrm{x}} \mathrm{Zn}_{0,2} \mathrm{Fe}_{2} \mathrm{O}_{4}\right.$, com $0 \leq \mathrm{x} \leq 0,4)$ preparadas pelo método "soft chemistry", reportou também o efeito do cobre no processo de densificação e sinterização das ferritas Ni-Zn. Neste trabalho, verificou-se que para a temperatura de sinterização $1000^{\circ} \mathrm{C}$ foi observada uma diminuição da magnetização de saturação de $68,6 \mathrm{emu} / \mathrm{g}$ para $66,3 \mathrm{emu} / \mathrm{g}$ e um aumento densidade de $4,69 \mathrm{~g} / \mathrm{cm}^{3}$ para $4,93 \mathrm{~g} / \mathrm{cm}^{3}$ para as concentrações 0,1 e 0,4 mol de $\mathrm{Cu}$, respectivamente. $\mathrm{O}$ aumento da densidade foi atribuído ao aumento do tamanho de grão e redução dos poros na microestrutura da ferrita. Entretanto, a condução aumentou para as composições $\mathrm{x} \geq 0,3$, o que foi atribuído à formação de segunda fase $\mathrm{CuO}$ e $\mathrm{CuFe}_{2} \mathrm{O}_{4}$ [11]. O efeito da substituição do cobre por zinco na ferrita de cobre com composição $\mathrm{Cu}_{1-\mathrm{x}} \mathrm{Zn}_{\mathrm{x}} \mathrm{Fe}_{2} \mathrm{O}_{4}, \mathrm{x}=$ 0,0/0,25/0,5/0,75/1,0 mol de cobre, sobre as propriedades magnéticas, porosidade, tamanho de grão e coercividade foi investigado [12]. Neste trabalho, as ferritas $\mathrm{Cu}_{1-\mathrm{x}} \mathrm{Zn}_{\mathrm{x}} \mathrm{Fe}_{2} \mathrm{O}_{4}$ foram preparadas pela técnica de reação do estado sólido usando $\mathrm{CuO}, \mathrm{ZnO}$ e $\mathrm{Fe}_{2} \mathrm{O}_{3}$ como reagentes. Foi observado que o tamanho de grão e a porosidade diminuem com a substituição do cobre pelo zinco. O aumento da concentração de zinco ocasionou um aumento em Hc, explicado pelo fato de $\mathrm{Hc}$ ser inversamente proporcional ao tamanho do grão [13]. Também foi observado um aumento da magnetização de saturação com a substituição do cobre pelo zinco, explicado pelo aumento da densidade geométrica [14, 15]. Foi avaliada a substituição do cobre no pó de ferrita Ni-Zn com composição nominal $\mathrm{Ni}_{(0,8-\mathrm{x})} \mathrm{Zn}_{0,2} \mathrm{Cu}_{\mathrm{x}} \mathrm{Fe}_{2} \mathrm{O}_{4}$, $(x=0,0 / 0,05 / 0,15 / 0,25 / 0,42 / 0,45 / 0,5 / 0,55 / 0,6$ mol de $\mathrm{Cu}$ ) preparada por co-precipitação química e sinterizada a $1000{ }^{\circ} \mathrm{C}$ [16]. Através de análise de difração de raios $\mathrm{X}$ observaram a formação da fase de espinélio cúbico para $\mathrm{x}=0,25$ e presença da segunda fase para composições com $\mathrm{x}>0,25$. Foi observado um tamanho do cristalito de $10 \mathrm{~nm}$ para o pó como preparado e $50 \mathrm{~nm}$ para as amostras calcinadas a $1000{ }^{\circ} \mathrm{C}$. O parâmetro de rede aumentou com a elevação do teor de cobre, com uma variação não-linear. Isto foi justificado em função do cobre estar presente em diferentes estados iônicos $\left(\mathrm{Cu}^{+} \mathrm{e} \mathrm{Cu}{ }^{2+}\right)$, os quais apresentam raios iônicos $\mathrm{Cu}^{+}(0,96 \AA)$ e $\mathrm{Cu}^{2+}(0,87 \AA)$ e em substituição aos íons de $\mathrm{Ni}^{2+}$ com raioiônico $0,83 \AA$, causa uma distribuição aleatória nos sítios $\mathrm{B}$, influenciando o parâmetro de rede. Uma redução na magnetização de saturação foi também observada pela substituição do níquel pelo cobre nos sítios $\mathrm{B}$ devido ao momento magnético do $\mathrm{Ni}^{2+}(2 \mu \mathrm{B})$ ser maior que o do $\mathrm{Cu}^{2+}(1 \mu \mathrm{B})$ e $\mathrm{Cu}^{+}(0 \mu \mathrm{B})$. Quanto ao campo coercitivo Hc observou-se uma dependência com o tamanho de cristalito, ou seja, houve um aumento até valores de 35 $\mathrm{nm}$, depois uma queda acentuada. Foi reportado o efeito do cobre nas propriedades eletromagnéticas das ferritas de $\mathrm{Mg}-\mathrm{Zn}-\mathrm{Cu}$ preparadas pelo método de auto-combustão $\left(\mathrm{Mg}_{0,5-\mathrm{x}} \mathrm{Cu}_{\mathrm{x}} \mathrm{Zn}_{0,5}\right)\left(\mathrm{Fe}_{2} \mathrm{O}_{3}\right)_{0,98} \quad(\mathrm{x}=0,2 / 0,25 / 0,30 / 0,35 / 0,4)$ sinterizadas a 900 e $9500^{\circ} \mathrm{C}$ por $4 \mathrm{~h}$ [17]. Foi mostrado que o cobre possui influência significativa nas propriedades eletromagnéticas, como permeabilidade inicial, resistividade, constante dielétrica e perdas dielétricas. Foi observado um aumento da densidade aparente, aumento no tamanho de grão, aumento na permeabilidade inicial e uma diminuição na resistividade elétrica com o aumento da concentração do cobre. Esta diminuição da resistividade elétrica com o incremento do cobre $\left(\mathrm{Cu}^{2+}\right)$ foi atribuída ao fato que, na ferrita $\mathrm{Mg}-\mathrm{Zn}-\mathrm{Cu}$, os sítios B estão ocupados por $\mathrm{Mg}^{2+}, \mathrm{Fe}^{2+}$ e $\mathrm{Cu}^{2+}$ e que com o aumento da temperatura de sinterização ocorre uma mudança nos estados de oxidação do $\mathrm{Cu}^{+}$para $\mathrm{Cu}^{2+}$. 
Este trabalho tem como objetivo a sinterização a $1000^{\circ} \mathrm{C}$ de pós de ferritas Ni-Zn dopadas com cobre de composição nominal $\mathrm{Ni}_{0,5-\mathrm{x}} \mathrm{Cu}_{\mathrm{x}} \mathrm{Zn}_{0,5} \mathrm{Fe}_{2} \mathrm{O}_{4} \quad(\mathrm{x}=0,1 / 0,2 / 0,3 / 0,4 \mathrm{~mol})$ previamente preparados por reação de combustão, com tamanho de cristalito entre 23 a $29 \mathrm{~nm}$, e subseqüente avaliação da estrutura, microestrutura e propriedade magnética das amostras sinterizadas, visando sua aplicação em dispositivos magnéticos moles ou intermediários.

\section{MATERIAIS E MÉTODOS}

Os materiais foram pós previamente preparados com tamanho de cristalito na faixa de 23 a $29 \mathrm{~nm}$, apresentado fase majoritária cristalina do espinélio inverso $\mathrm{Ni}_{0,5-\mathrm{x}} \mathrm{Cu}_{\mathrm{x}} \mathrm{Zn}_{0,5} \mathrm{Fe}_{2} \mathrm{O}_{4}$ e presença de traços de fases secundárias como $\mathrm{Fe}_{2} \mathrm{O}_{3}$ e $\mathrm{CuO}$. $\mathrm{O}$ tamanho de partícula determinada a partir da área superficial por BET estava na faixa de 26 a $64 \mathrm{~nm}$ [18]. Os sistemas avaliados foram designados $\mathrm{R} 01\left(\mathrm{Ni}_{0,4} \mathrm{Cu}_{0,1} \mathrm{Zn}_{0,5} \mathrm{Fe}_{2} \mathrm{O}_{4}\right), \mathrm{R} 02$ $\left(\mathrm{Ni}_{0,3} \mathrm{Cu}_{0,2} \mathrm{Zn}_{0,5} \mathrm{Fe}_{2} \mathrm{O}_{4}\right), \mathrm{R} 03\left(\mathrm{Ni}_{0,2} \mathrm{Cu}_{0,3} \mathrm{Zn}_{0,5} \mathrm{Fe}_{2} \mathrm{O}_{4}\right)$ e $\mathrm{R} 04$ $\left(\mathrm{Ni}_{0,1} \mathrm{Cu}_{0,4} \mathrm{Zn}_{0,5} \mathrm{Fe}_{2} \mathrm{O}_{4}\right)$. Os pós dos sistemas foram granulados em uma peneira de nylon de malha $100(150 \mu \mathrm{m})$. As amostras foram conformadas por prensagem uniaxial de $385 \mathrm{MPa}$ na forma de pastilhas com 9,8 mm de diâmetro e aproximadamente $3 \mathrm{~mm}$ de espessura. As amostras foram sinterizadas ao ar a $1000{ }^{\circ} \mathrm{C} / 2 \mathrm{~h}$ e taxa de $10{ }^{\circ} \mathrm{C} / \mathrm{min}$. As medidas de densidade aparente $(\mathrm{Da})$ e porosidade aparente $(\mathrm{Pa})$ das amostras sinterizadas foram determinadas pelo método de imersão utilizando o princípio de Archimedes (norma ABNT-NBR6620). A caracterização microestrutural foi feita por microscopia eletrônica de varredura (MEV) no equipamento JEOL, modelo JSM-5900 e a determinação do tamanho médio de grão foi feita com o programa Mocha (Jandell, analisador de imagens) fazendo-se a contagem média de 200 grãos por amostra. Os parâmetros magnéticos (campo coercitivo Hc, magnetização de saturação Ms e perdas por histerese $\mathrm{P}_{\mathrm{H}}$ ) foram determinados através curva de histerese $\mathrm{M}-\mathrm{H}$. Os ciclos de histerese magnética foram obtidos em magnetômetro de amostra vibrante com campo máximo aplicado de $20 \mathrm{kOe}$. A determinação das fases nas amostras sinterizadas foi feita em um difratômetro de raios X Shimadzu modelo XRD 6000, com radiação $\mathrm{CuK}$ com monocromador secundário de grafite curvo na faixa $2 \theta$ de 20 a $75^{\circ}$, modo contínuo, $3^{\circ} / \mathrm{min}$. O tamanho médio de cristalito foi calculado a partir da largura dos picos de maior intensidade $\left(\mathrm{d}_{111}, \mathrm{~d}_{022}, \mathrm{~d}_{113}, \mathrm{~d}_{222}, \mathrm{~d}_{004}, \mathrm{~d}_{224}, \mathrm{~d}_{333}, \mathrm{e}_{044}\right)$ corrigido pelo alargamento instrumental obtido a partir de uma amostra de silício policristalino (utilizado como padrão) com a equação de Scherrer [19]. Os parâmetros de rede foram obtidos através de rotina disponível no pacote de programas FullProff [20].

\section{RESULTADOS E DISCUSSÃO}

A Fig. 1 apresenta os difratogramas de raios $\mathrm{X}$ para os sistemas R01, R02, R03 e R04 sinterizados a $1000^{\circ} \mathrm{C} / 2 \mathrm{~h}$. Em $\mathrm{Ni}_{0,3} \mathrm{Cu}_{0,2} \mathrm{Zn}_{0,5} \mathrm{Fe}_{2} \mathrm{O}_{4}$ e $\mathrm{Ni}_{0,2} \mathrm{Cu}_{0,3} \mathrm{Zn}_{0,5} \mathrm{Fe}_{2} \mathrm{O}_{4}$ houve a formação

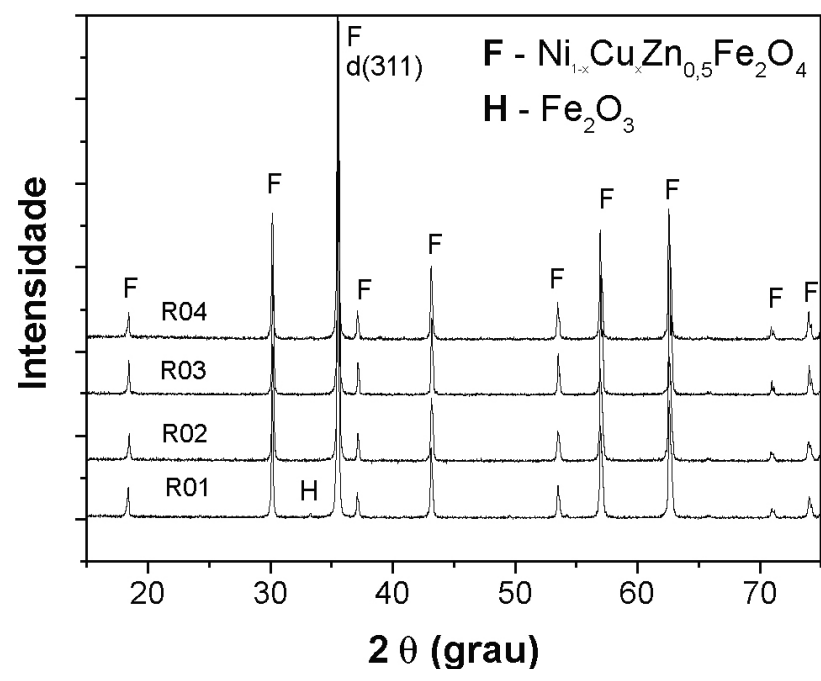

Figura 1: Difratogramas de raios $X$ dos sistemas $\mathrm{Ni}_{0,4} \mathrm{Cu}_{0,1} \mathrm{Zn}_{0,5} \mathrm{Fe}_{2} \mathrm{O}_{4}$, $\mathrm{Ni}_{0,3} \mathrm{Cu}_{0,2} \mathrm{Zn}_{0,5} \mathrm{Fe}_{2} \mathrm{O}_{4}, \quad \mathrm{Ni}_{0,2} \mathrm{Cu}_{0,3} \mathrm{Zn}_{0,5} \mathrm{Fe}_{2} \mathrm{O}_{4}$ e $\mathrm{Ni}_{0,1} \mathrm{Cu}_{0,4} \mathrm{Zn}_{0,5} \mathrm{Fe}_{2} \mathrm{O}_{4}$ sinterizados a $1000^{\circ} \mathrm{C} / 2 \mathrm{~h}$.

[Figure 1: X-ray diffraction patterns of the $\mathrm{Ni} \mathrm{Cu}_{0.4} \mathrm{Cu}_{0.1} \mathrm{Zn}_{0.5} \mathrm{Fe}_{2} \mathrm{O}_{4}$, $\mathrm{Ni}_{0.3} \mathrm{Cu}_{0.2} \mathrm{Zn}_{0.5} \mathrm{Fe}_{2} \mathrm{O}_{4}, \mathrm{Ni}_{0.2} \mathrm{Cu}_{0.3} \mathrm{Zn}_{0.5} \mathrm{Fe}_{2} \mathrm{O}_{4}$ and $\mathrm{Ni}_{0.1} \mathrm{Cu}_{0.4} \mathrm{Zn}_{0.5} \mathrm{Fe}_{2} \mathrm{O}_{4}$ specimens sintered at $1000^{\circ} \mathrm{C} / 2 \mathrm{~h}$.]

completa da fase cristalina da ferrita $\mathrm{Ni}_{1-\mathrm{x}} \mathrm{Cu}_{\mathrm{x}} \mathrm{Zn}_{0,5} \mathrm{Fe}_{2} \mathrm{O}_{4}$ ( $\mathrm{x}$ $=0,1 / 0,2 / 0,3 / 0,4 \mathrm{~mol}$ de cobre) com estrutura cúbica do espinélio inverso (JCPDF 48-0489). Todavia, para o sistema $\mathrm{Ni}_{0,4} \mathrm{Cu}_{0,1} \mathrm{Zn}_{0,5} \mathrm{Fe}_{2} \mathrm{O}_{4}$ e $\mathrm{Ni}_{0,1} \mathrm{Cu}_{0,4} \mathrm{Zn}_{0,5} \mathrm{Fe}_{2} \mathrm{O}_{4}$ foram observados, além da fase majoritária da ferrita, traços da segunda fase identificada como $\mathrm{Fe}_{2} \mathrm{O}_{3}$, hematita, (JCPDF 25-1228)

A Fig. 2 mostra a variação do parâmetro de rede e do tamanho do cristalito com o aumento da concentração de cobre.
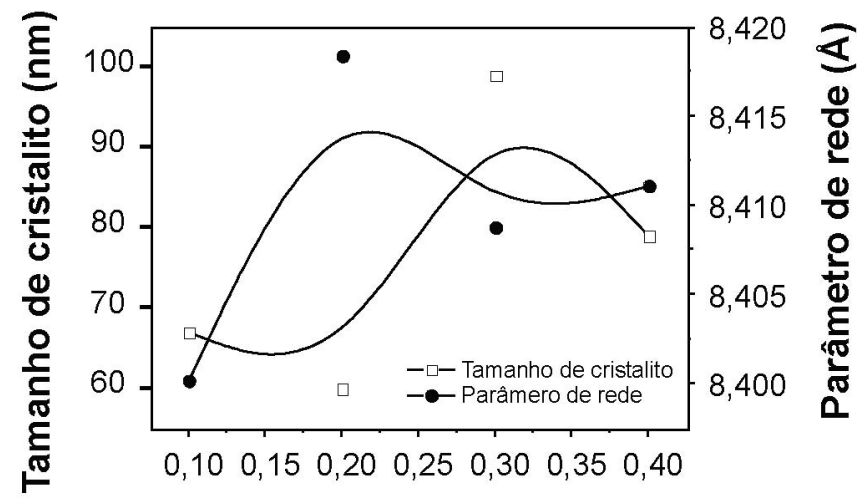

\section{Concentração de cobre (mol)}

Figura 2: Parâmetro de rede e tamanho de cristalito em função da concentração de cobre.

[Figure 2: Lattice parameter and crystallite size as a function of copper concentration.]

Existe uma tendência de crescimento do parâmetro de rede, pois só a amostra com concentração $0,20 \mathrm{~mol} \mathrm{de} \mathrm{Cu}^{2+}$ apresentou um excessivo aumento em relação as demais concentrações. Este comportamento não linear foi semelhante 
Tabela I - Características físicas das amostras sinterizadas a $1000^{\circ} \mathrm{C} / 2 \mathrm{~h}$ com taxa de aquecimento $10^{\circ} \mathrm{C} / \mathrm{min}$. [Table I - Physical characteristics of the specimens sintered at $1000^{\circ} \mathrm{C} / 2 \mathrm{~h}$ with heating rate $10{ }^{\circ} \mathrm{C} / \mathrm{min}$.]

\begin{tabular}{cccccccc}
\hline \multicolumn{1}{c}{ Sistemas } & $\begin{array}{c}\mathrm{Dv} \\
\left(\mathrm{g} / \mathrm{cm}^{3}\right)\end{array}$ & $\begin{array}{c}\mathrm{Dv} / \mathrm{Dt} \\
(\%)\end{array}$ & $\begin{array}{c}\mathrm{Dg} \\
\left(\mathrm{g} / \mathrm{cm}^{3}\right)\end{array}$ & $\begin{array}{c}\mathrm{Dg} / \mathrm{Dt} \\
(\%)\end{array}$ & $\begin{array}{c}\mathrm{Da} \\
\left(\mathrm{g} / \mathrm{cm}^{3}\right)\end{array}$ & $\begin{array}{c}\mathrm{Da} / \mathrm{Dt} \\
(\%)\end{array}$ & $\begin{array}{c}\mathrm{Pa} \\
(\%)\end{array}$ \\
\hline $\mathrm{Ni}_{0,4} \mathrm{Cu}_{0,1} \mathrm{Zn}_{0,5} \mathrm{Fe}_{2} \mathrm{O}_{4}$ & 2,95 & 56,33 & 3,46 & 66,1 & 4,15 & 79,2 & 33,8 \\
$\mathrm{Ni}_{0,3} \mathrm{Cu}_{0,2} \mathrm{Zn}_{0,5} \mathrm{Fe}_{2} \mathrm{O}_{4}$ & 2,90 & 55,37 & 3,89 & 74,3 & 4,16 & 79,4 & 21,3 \\
$\mathrm{Ni}_{0,2} \mathrm{Cu}_{0,3} \mathrm{Zn}_{0,5} \mathrm{Fe}_{2} \mathrm{O}_{4}$ & 2,88 & 55,00 & 3,46 & 66,1 & 4,07 & 77,7 & 11,3 \\
$\mathrm{Ni}_{0,1} \mathrm{Cu}_{0,4} \mathrm{Zn}_{0,5} \mathrm{Fe}_{2} \mathrm{O}_{4}$ & 2,90 & 55,37 & 3,59 & 68,6 & 4,44 & 84,8 & 6,7 \\
\hline Densidade teórica (Dt) $=5,237{\mathrm{~g} / \mathrm{cm}^{3}(J C P D F}^{2}$ 48-0489) $-\mathrm{Cu}_{0,1} \mathrm{Fe}_{1,9} \mathrm{Ni}_{0,6} \mathrm{Zn}_{0,35} \mathrm{O}_{4}$ & & &
\end{tabular}

ao observado no efeito do cobre nas propriedades magnéticas de ferritas $\mathrm{Ni}-\mathrm{Zn}$ dopadas com cobre pelo método químico de cooprecipitação, que foi explicado pela mudança nos estados de oxidação de $\mathrm{Cu}^{2+}$ para $\mathrm{Cu}^{+}$nos sítios octáedricos [16]. Este mesmo efeito também foi observado em ferritas de Mg-Zn obtidas pelo método de auto-combustão e foi justificado pelo fato de que durante a sinterização os íons $\mathrm{Cu}^{2+}$ podem estar se oxidando para $\mathrm{Ce}^{1+}[17]$. Como os raios iônicos são diferentes, porém maiores que o raio iônico do $\mathrm{Ni}^{2}+(0,83 \AA)$, estes aumentam o parâmetro de rede com o aumento do teor de cobre em substituição aos íons níquel de forma não-linear, com uma distribuição aleatória. Quanto ao tamanho de cristalito, podemos observar um comportamento aleatório, o qual pode também ter sido influenciado pela mudança no estado de oxidação dos íons cobre, visto que esta mudança pode influenciar a formação de fases secundárias, como observado para os sistemas $\mathrm{Ni}_{0,4} \mathrm{Cu}_{0,1} \mathrm{Zn}_{0,5} \mathrm{Fe}_{2} \mathrm{O}_{4}$ e $\mathrm{Ni}_{0,1} \mathrm{Cu}_{0,4} \mathrm{Zn}_{0,5} \mathrm{Fe}_{2} \mathrm{O}_{4}$, o que influencia o tamanho de cristalito.

A Tabela I mostra os resultados das características físicas (densidade geométrica, densidade a verde e as respectivas densidades relativas) dos sistemas antes e após sinterização como também a densidade aparente e a porosidade aparente.

A densidade a verde não apresentou mudanças significativas com o aumento da concentração de cobre. Os valores de densidade a verde relativa foram superiores a $50 \%$. Este resultado mostra que para todos os sistemas estudados houve uma boa densidade de compactação, justificado porque todos os sistemas estudados são compostos por partículas nanométricas aglomeradas. Estes aglomerados formados são do tipo mole constituídos por forças fracas de Van der Waals característicos de pós de ferritas preparados por reação de combustão [21-23]. Tais aglomerados moles de nanopartículas são de tamanhos $<5 \mu \mathrm{m}$, o que durante a compactação conseguem se arranjar de forma adequada resultando em bons valores de densidade a verde. A ferrita $\mathrm{Ni}-\mathrm{Zn}$ (JCPDS 52-0278) possui densidade teórica 5,361 $\mathrm{g} / \mathrm{cm}^{3}$, enquanto a ferrita Cu-Zn (JCPDS 77-0012) 5,406 $\mathrm{g} / \mathrm{cm}^{3}$; isto resulta em uma variação na densidade de $8,3 \%$ quando substitui totalmente os íons de $\mathrm{Ni}^{2+}$ por $\mathrm{Cu}^{2+}$. Então, de acordo com o aumento da substituição dos íons de $\mathrm{Ni}^{2+}$ por $\mathrm{Cu}^{2+}$ é esperado um aumento na densidade teórica das amostras sinterizadas a $1000{ }^{\circ} \mathrm{C}$. Na Tabela I, de uma forma geral, observa-se um leve aumento na densidade geométrica e aparente, e uma redução da porosidade aparente com o aumento da concentração de cobre. Com relação à densidade geométrica, observou-se uma densidade relativa de 74,3\% para o sistema $\mathrm{Ni}_{0,3} \mathrm{Cu}_{0,2} \mathrm{Zn}_{0,5} \mathrm{Fe}_{2} \mathrm{O}_{4}$, superior aos valores de densidade geométrica determinadas para os demais sistemas. Isto pode ser justificado pela precisão nas medidas altura e diâmetro das amostras. Por outro lado, para a medida densidade aparente e porosidade aparente determinada pelo método de Arquimedes, observou-se maior precisão nos resultados, sendo o maior valor de densidade relativa alcançado para o sistema $\mathrm{Ni}_{0,1} \mathrm{Cu}_{0,4} \mathrm{Zn}_{0,5} \mathrm{Fe}_{2} \mathrm{O}_{4}, 84,8 \%$. Houve uma redução significativa na porosidade aparente de $33,8 \%$ $\left(\mathrm{Ni}_{0,4} \mathrm{Cu}_{0,1} \mathrm{Zn}_{0,5} \mathrm{Fe}_{2} \mathrm{O}_{4}\right)$ para 6,7\% $\left(\mathrm{Ni}_{0,1} \mathrm{Cu}_{0,4} \mathrm{Zn}_{0,5} \mathrm{Fe}_{2} \mathrm{O}_{4}\right)$. Analisando os resultados de densidade aparente determinados com o valor teórico para a ferrita $\mathrm{Cu}_{0,1} \mathrm{Fe}_{1,9} \mathrm{Ni}_{0,6} \mathrm{Zn}_{0,35} \mathrm{O}_{4}$ sinterizada a $1250{ }^{\circ} \mathrm{C}$, podemos observar que as amostras dopadas com cobre apresentaram uma redução na densidade após sinterização a $1000{ }^{\circ} \mathrm{C}$ variando entre $15,2 \%$ e $22,2 \%$, o que pode ser considerada baixa visto a diferença na temperatura de sinterização. Além disso, podemos também justificar que a temperatura de sinterização de $1000^{\circ} \mathrm{C} / 2 \mathrm{~h}$ foi suficiente para causar inicio da evaporação do cobre na forma de óxido de cobre, que funde a $1026^{\circ} \mathrm{C}$, reduzindo assim a densidade. Por outro lado, o cobre em fase líquida tende a segregar para o contorno de grão, diminuindo a porosidade total das amostras sinterizadas nesta temperatura.

As Figs. 3a, b, c e d mostram as micrografias obtidas por microscopia eletrônica de varredura dos sistemas R01, R02, R03 e R04 após sinterização a $1000{ }^{\circ} \mathrm{C} / 2 \mathrm{~h}$ com taxa de aquecimento $10^{\circ} \mathrm{C} / \mathrm{min}$. Observou-se aumento no tamanho dos grãos com o aumento na concentração de cobre em $\mathrm{Ni}_{1-\mathrm{x}} \mathrm{Cu}_{\mathrm{x}} \mathrm{Zn}_{0,5} \mathrm{Fe}_{2} \mathrm{O}_{4}$ e que o tratamento de $1000{ }^{\circ} \mathrm{C} / 2 \mathrm{~h}$ não foi suficiente para fornecer a força motriz para completa formação da microestrutura. Para todos os sistemas estudados foi observada uma microestrutura heterogênea, com grãos de formato irregular e poros intergranulares. Verifica-se também um aumento no tamanho de poro, principalmente quando comparamos as microestruturas do sistema $\mathrm{Ni}_{0,4} \mathrm{Cu}_{0,1} \mathrm{Zn}_{0,5} \mathrm{Fe}_{2} \mathrm{O}_{4}$ com a do sistema $\mathrm{Ni}_{0,1} \mathrm{Cu}_{0,4} \mathrm{Zn}_{0,5} \mathrm{Fe}_{2} \mathrm{O}_{4}$. 

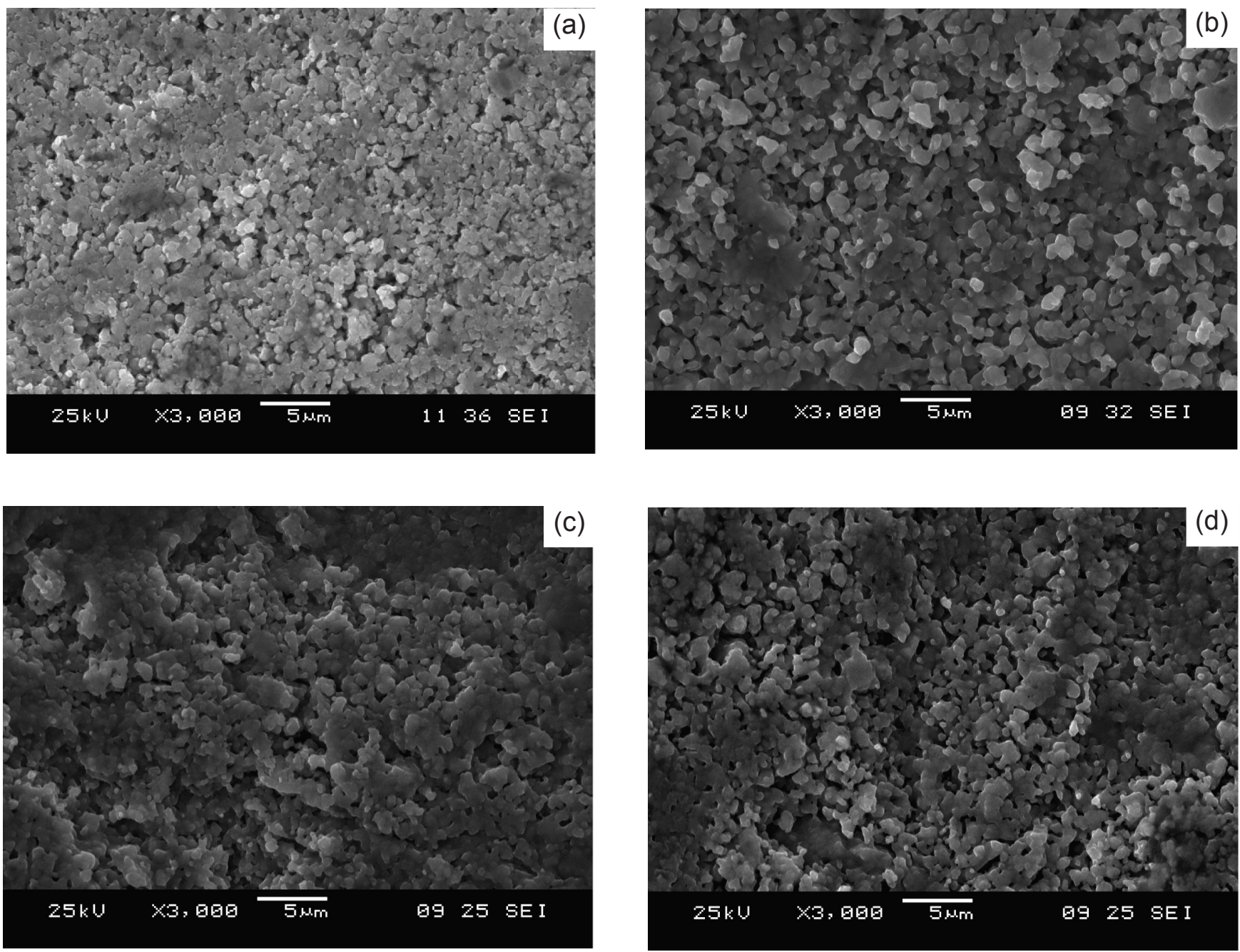

Figura 3: Micrografias obtidas por microscopia eletrônica de varredura para os sistemas sinterizados a $1000^{\circ} \mathrm{C} / 2 \mathrm{~h}$ com taxa de aquecimento $10{ }^{\circ} \mathrm{C} /$ min. (a) $\mathrm{Ni}_{0,4} \mathrm{Cu}_{0,1} \mathrm{Zn}_{0,5} \mathrm{Fe}_{2} \mathrm{O}_{4}$, (b) $\mathrm{Ni}_{0,3} \mathrm{Cu}_{0,2} \mathrm{Zn}_{0,5} \mathrm{Fe}_{2} \mathrm{O}_{4}$, (c) $\mathrm{Ni}_{0,2} \mathrm{Cu}_{0,3} \mathrm{Zn}_{0,5} \mathrm{Fe}_{2} \mathrm{O}_{4} \mathrm{e}$ (d) $\mathrm{Ni}_{0,1} \mathrm{Cu}_{0,4} \mathrm{Zn}_{0,5} \mathrm{Fe}_{2} \mathrm{O}_{4}$.

[Figure 3: SEM micrographs of the specimens sintered at $1000{ }^{\circ} \mathrm{C} / 2 \mathrm{~h}$ with heating rate $10^{\circ} \mathrm{C} / \mathrm{min}$ (a) $\mathrm{Ni}_{0.4} \mathrm{Cu} \mathrm{u}_{0.1} \mathrm{Zn} \mathrm{n}_{0.5} \mathrm{Fe} \mathrm{O}_{2} \mathrm{O}_{4}$ (b) $\mathrm{Ni}_{0.3} \mathrm{Cu}_{0.2} \mathrm{Zn}_{0.5} \mathrm{Fe}_{2} \mathrm{O}_{4}$, (c) $\mathrm{Ni}_{0.2} \mathrm{Cu}_{0.3} \mathrm{Zn}_{0.5} \mathrm{Fe}_{2} \mathrm{O}_{4}$ and (d) $\mathrm{Ni}_{0.1} \mathrm{Cu}_{0.4} \mathrm{Zn}_{0.5} \mathrm{Fe}_{2} \mathrm{O}_{4}$. J

Tabela II - Tamanho médio de grãos e de poros intergranulares para os sistemas $\mathrm{Ni}_{0,4} \mathrm{Cu}_{0,1} \mathrm{Zn}_{0,5} \mathrm{Fe}_{2} \mathrm{O}_{4}, \mathrm{Ni}_{0,3} \mathrm{Cu}_{0,2} \mathrm{Zn}_{0,5} \mathrm{Fe}_{2} \mathrm{O}_{4}$, $\mathrm{Ni}_{0,2} \mathrm{Cu}_{0,3} \mathrm{Zn}_{0,5} \mathrm{Fe}_{2} \mathrm{O}_{4}$ e $\mathrm{Ni}_{0,1} \mathrm{Cu}_{0,4} \mathrm{Zn}_{0,5} \mathrm{Fe}_{2} \mathrm{O}_{4}$ sinterizados a $1000{ }^{\circ} \mathrm{C} / 2 \mathrm{~h}$ com taxa de aquecimento $10^{\circ} \mathrm{C} / \mathrm{min}$.

[Table II -Average grain size and intergranularpore size of the $\mathrm{Ni}_{0.4} \mathrm{Cu}_{0.1} \mathrm{Zn}_{0.5} \mathrm{Fe}_{2} \mathrm{O}_{4}, \mathrm{Ni}_{0.3} \mathrm{Cu} u_{0.2} \mathrm{Zn}_{0.5} \mathrm{Fe}_{2} \mathrm{O}_{4}, \mathrm{Ni}_{0.2} \mathrm{Cu}_{0.3} \mathrm{Zn}_{0.5} \mathrm{Fe}_{2} \mathrm{O}_{4}$ and $\mathrm{Ni}_{0.1} \mathrm{Cu}_{0.4} \mathrm{Zn}_{0.5} \mathrm{Fe}_{2} \mathrm{O}_{4}$ specimens sintered at $1000^{\circ} \mathrm{C} / 2 \mathrm{~h}$ with heating rate $10^{\circ} \mathrm{C} / \mathrm{min}$.]

\begin{tabular}{ccc}
\hline Amostra & $\begin{array}{c}\text { Tamanho de } \\
\text { grão }(\mu \mathrm{m})\end{array}$ & $\begin{array}{c}\text { Tamanho de } \\
\text { poro }(\mu \mathrm{m})\end{array}$ \\
\hline $\mathrm{Ni}_{0,4} \mathrm{Cu}_{0,1} \mathrm{Zn}_{0,5} \mathrm{Fe}_{2} \mathrm{O}_{4}$ & $0,65 \pm 0,30$ & $0,60 \pm 0,33$ \\
$\mathrm{Ni}_{0,3} \mathrm{Cu}_{0,2} \mathrm{Zn}_{0,5} \mathrm{Fe}_{2} \mathrm{O}_{4}$ & $0,76 \pm 0,32$ & $0,73 \pm 0,33$ \\
$\mathrm{Ni}_{0,2} \mathrm{Cu}_{0,3} \mathrm{Zn}_{0,5} \mathrm{Fe}_{2} \mathrm{O}_{4}$ & $0,55 \pm 0,30$ & $0,64 \pm 0,32$ \\
$\mathrm{Ni}_{0,1} \mathrm{Cu}_{0,4} \mathrm{Zn}_{0,5} \mathrm{Fe}_{2} \mathrm{O}_{4}$ & $0,68 \pm 0,27$ & $0,76 \pm 0,32$ \\
\hline
\end{tabular}

A Tabela II mostra os resultados do tamanho médio de grão e de poros intergranulares obtidos através de várias micrografias. Foi construído histograma com o intuito de se avaliar a microestrutura por meio de uma análise semi-quantitativa com uma qualitativa fornecida pelas micrografias na Fig. 3. De uma forma geral, podemos observar que o aumento da concentração de cobre favoreceu o aumento do tamanho médio de grãos e do tamanho de poros intergranulares. Pela Tabela I a porosidade aparente diminui com a adição do cobre, ou seja, a porosidade total diminui, porém os poros ficam maiores em tamanho. Podemos observar que o aumento da concentração de cobre favoreceu um aumento do tamanho de grão de aproximadamente 4,6\% comparando o sistema $\mathrm{Ni}_{0,4} \mathrm{Cu}_{0,1} \mathrm{Zn}_{0,5} \mathrm{Fe}_{2} \mathrm{O}_{4}$ com o sistema $\mathrm{Ni}_{0,1} \mathrm{Cu}_{0,4} \mathrm{Zn}_{0,5} \mathrm{Fe}_{2} \mathrm{O}_{4}$. A porosidade intergranular também aumentou com o aumento da concentração de cobre. Houve um aumento de $26,7 \%$ comparando o sistema $\mathrm{Ni}_{0,4} \mathrm{Cu}_{0,1} \mathrm{Zn}_{0,5} \mathrm{Fe}_{2} \mathrm{O}_{4}$ com o $\mathrm{Ni}_{0,1} \mathrm{Cu}_{0,4} \mathrm{Zn}_{0,5} \mathrm{Fe}_{2} \mathrm{O}_{4}$. 

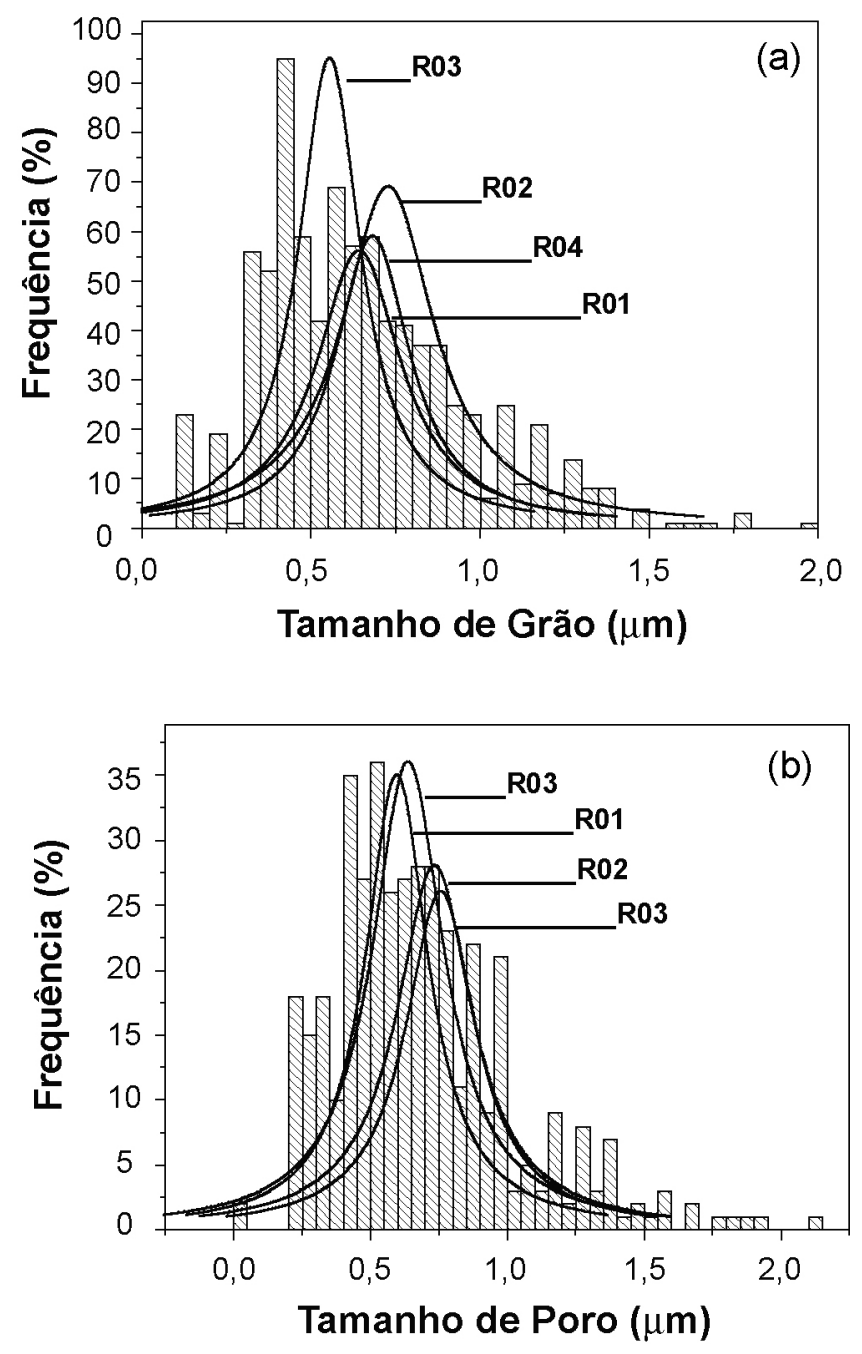

Figura 4: Histogramas e curvas de distribuição de Lorentz de tamanho de poros dos sistemas R01, R02, R03 e R04 sinterizados a $1000{ }^{\circ} \mathrm{C} / 2 \mathrm{~h}$ com taxa de aquecimento $10{ }^{\circ} \mathrm{C} / \mathrm{min}$. (a) tamanho de grão e (b) tamanho de poro.

[Figure 4: Histograms and Lorentz distribution curves of pore sizes of the specimens sintered at $1000{ }^{\circ} \mathrm{C} / 2 \mathrm{~h}$ with heating rate 10 ${ }^{\circ} \mathrm{C} / \mathrm{min}$. (a) grain size and (b) pore size.]

As Figs. 4a e 4b mostram os histogramas e curvas de distribuição Lorentz para o tamanho de grãos e tamanho de poros para os sistemas R01, R02, R03 e R04. Observa-se uma microestrutura com tamanho de grãos menos heterogênea (distribuição mais estreita) para o sistema $\mathrm{Ni}_{0,2} \mathrm{Cu}_{0,3} \mathrm{Zn}_{0,5} \mathrm{Fe}_{2}$ $\mathrm{O}_{4}$ e uma microestrutura mais heterogênea (distribuição mais larga) para o sistema $\mathrm{Ni}_{0,3} \mathrm{Cu}_{0,2} \mathrm{Zn}_{0,5} \mathrm{Fe}_{2} \mathrm{O}_{4}$. As curvas de distribuição de Lorentz (Fig. 4b) mostram que todos os sistemas estudados apresentaram uma distribuição dos tamanhos de poros muito larga, com grande heterogeneidade neste parâmetro.

A Fig. 5 apresenta a dependência da magnetização $(\sigma)$ com o campo magnético aplicado $(\mathrm{H})$ por meio de uma curva ou laço de histerese $\boldsymbol{\sigma}-\boldsymbol{H}$ para os sistemas R01, R02, R02 e R04. Foi possível determinar os valores de alguns parâmetros magnéticos, tais como campo coercitivo (Hc), magnetização remanente ( $\mathrm{Mr}$ ou or) e magnetização de saturação (Ms ou $\sigma s$ ). A magnetização de saturação foi determinada fazendo um ajuste dos dados do campo aplicado para a função $\sigma=\sigma s(1-\alpha / H)$, onde $\sigma$ é a magnetização, $\sigma s$ é a magnetização de saturação, $\alpha$ é o parâmetro do ajuste e

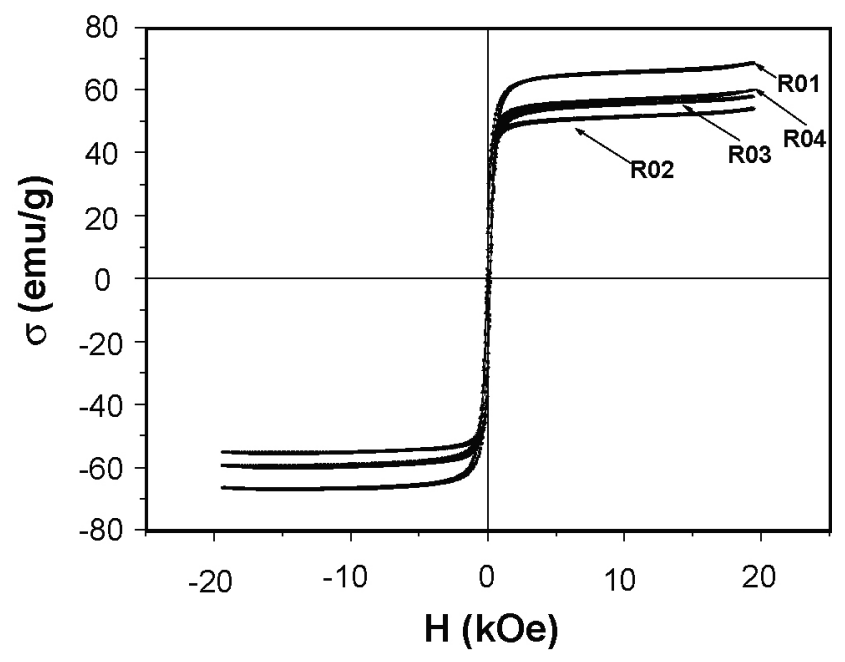

Figura 5: Curvas de histerese magnética dos sistemas $\mathrm{Ni}_{0,4} \mathrm{Cu}_{0,1} \mathrm{Zn}_{0,5} \mathrm{Fe}_{2} \mathrm{O}_{4}, \quad \mathrm{Ni}_{0,3} \mathrm{Cu}_{0,2} \mathrm{Zn}_{0,5} \mathrm{Fe}_{2} \mathrm{O}_{4}, \quad \mathrm{Ni}_{0,2} \mathrm{Cu}_{0,1} \mathrm{Zn}_{0,5} \mathrm{Fe}_{2} \mathrm{O}_{4}$ e $\mathrm{Ni}_{0,1} \mathrm{Cu}_{0,4} \mathrm{Zn}_{0,5} \mathrm{Fe}_{2} \mathrm{O}_{4}$ sinterizados a $1000^{\circ} \mathrm{C} / 2 \mathrm{~h}$.

[Figure 5: Magnetic hysteresis loops of $\mathrm{Ni}_{04} \mathrm{Cu}_{01} \mathrm{Zn}_{05} \mathrm{Fe}_{2} \mathrm{O}_{4}$, $\mathrm{Ni}_{0.3} \mathrm{Cu}_{0.2} \mathrm{Zn}_{0.5} \mathrm{Fe}_{2} \mathrm{O}_{4}, \mathrm{Ni} \mathrm{Cu}_{0.2} \mathrm{Cu}_{0.3} \mathrm{Zn}_{0.5} \mathrm{Fe}_{2} \mathrm{O}_{4}$ and $\mathrm{Ni} \mathrm{Ci}_{0.1} \mathrm{Cu}_{0.4} \mathrm{Zn}_{0.5} \mathrm{Fe}_{2} \mathrm{O}_{4}$ specimens sintered at $1000^{\circ} \mathrm{C} / 2 \mathrm{~h}$.]

H é o campo aplicado. Por meio das histereses observou-se a características de material magneticamente mole para os sistemas R01, R02, R02 e R04.

A magnetização e a permeabilidade aumentam pela facilidade do deslocamento reversível da parede dos domínios, e, para ocorrer esse deslocamento é necessário que a energia do campo externo aplicado seja maior que a energia de fixação da parede. Assim, quanto maior o tamanho de grão, menor será a área de contorno de grão e mais fácil será o deslocamento da parede de domínios; conseqüentemente, maior será a magnetização e a permeabilidade. Então, tanto a magnetização como a permeabilidade apresenta uma dependência direta com o tamanho de grão. A porosidade intergranular (no contorno de grão) também é indesejável, pois esta também impede o movimento da parede de domínios comprometendo essas propriedades. As perdas por histerese são menores para ferritas de grãos maiores, devido à dependência da coercividade com o tamanho de grão, ou seja, maior tamanho de grão, menor força coercitiva [24, 25]. Porém, deve-se ressaltar também que a magnetização e a permeabilidade dependem da característica intrínseca do material, sendo, portanto, dependentes da composição e distribuição dos cátions na rede espinélio.

Os resultados da medida dos parâmetros de histerese dos sistemas R01, R02, R03 e R04 obtidos a partir das curvas de 
TabelaIII-Parâmetros magnéticos dos sistemas $\mathrm{Ni}_{0.4} \mathrm{Cu}_{0,1} \mathrm{Zn}_{0.5} \mathrm{Fe}_{2} \mathrm{O}_{4}, \mathrm{Ni}_{0,3} \mathrm{Cu}_{0,2} \mathrm{Zn}_{0,5} \mathrm{Fe}_{2} \mathrm{O}_{4}$, $\mathrm{Ni}_{0,2} \mathrm{Cu}_{0,3} \mathrm{Zn}_{0,5} \mathrm{Fe}_{2} \mathrm{O}_{4}$ e $\mathrm{Ni}_{0,1} \mathrm{Cu}_{0,4} \mathrm{Zn}_{0,5} \mathrm{Fe}_{2} \mathrm{O}_{4}$ sinterizados a $1000{ }^{\circ} \mathrm{C} / 2 \mathrm{~h}$ com taxa de aquecimento $10{ }^{\circ} \mathrm{C} / \mathrm{min}$.

[Table III - Magnetic parameters of the $\mathrm{Ni}_{0.4} \mathrm{Cu}_{0.1} \mathrm{Zn}_{0.5} \mathrm{Fe}_{2} \mathrm{O}_{4}, \mathrm{Ni}_{0.3} \mathrm{Cu}_{0.2} \mathrm{Zn}_{0.5} \mathrm{Fe}_{2} \mathrm{O}_{4}, \mathrm{Ni}_{0.2}$ $\mathrm{Cu}_{0.3} \mathrm{Zn}_{0.5} \mathrm{Fe}_{2} \mathrm{O}_{4}$ and $\mathrm{Ni}_{0.1} \mathrm{Cu}_{0.4} \mathrm{Zn}_{0.5} \mathrm{Fe}_{2} \mathrm{O}_{4}$ specimens sintered at $1000^{\circ} \mathrm{C} / 2 \mathrm{~h}$ with heating rate $10^{\circ} \mathrm{C} / \mathrm{min}$.]

\begin{tabular}{cccccc}
\hline Sistemas & $\begin{array}{c}\mathrm{Ms} \\
(\mathrm{emu} / \mathrm{g})\end{array}$ & $\begin{array}{c}\mathrm{Mr} \\
(\mathrm{emu} / \mathrm{g})\end{array}$ & $\begin{array}{c}\mathrm{Hc} \\
(\mathrm{Oe})\end{array}$ & $\mathrm{Ms} / \mathrm{Mr}$ & $\begin{array}{c}\text { Área da curva } \\
(\mathrm{emu} / \mathrm{g} \text { x ke })\end{array}$ \\
\hline $\mathrm{Ni}_{0,4} \mathrm{Cu}_{0,1} \mathrm{Zn}_{0,5} \mathrm{Fe}_{2} \mathrm{O}_{4}$ & 68,79 & 22,74 & 102 & 3,02 & 1203,58 \\
$\mathrm{Ni}_{0,3} \mathrm{Cu}_{0,2} \mathrm{Zn}_{0,5} \mathrm{Fe}_{2} \mathrm{O}_{4}$ & 54,31 & 15,08 & 96 & 3,6 & 951,43 \\
$\mathrm{Ni}_{0,2} \mathrm{Cu}_{0,3} \mathrm{Zn}_{0,5} \mathrm{Fe}_{2} \mathrm{O}_{4}$ & 58,00 & 17,35 & 56 & 3,34 & 1014,84 \\
$\mathrm{Ni}_{0,1} \mathrm{Cu}_{0,4} \mathrm{Zn}_{0,5} \mathrm{Fe}_{2} \mathrm{O}_{4}$ & 60,24 & 16,03 & 81 & 3,75 & 1043,44 \\
\hline
\end{tabular}

histerese na Fig. 5 estão mostrados na Tabela III.

Houve uma pequena diminuição do valor da magnetização de saturação com o acréscimo do cobre no sistema $\left(\mathrm{Ni}_{1-\mathrm{x}} \mathrm{Cu}_{\mathrm{x}} \mathrm{Zn}_{0,5} \mathrm{Fe}_{2} \mathrm{O}_{4}\right)$. Isto já era esperado teoricamente de acordo com a distribuição dos cátions na rede espinélio. Além do mais, verificamos que o aumento da concentração de cobre aumenta o tamanho do poro intergranular, o qual tende a segurar o movimento da parede de domínios, contribuindo também para uma redução da magnetização de saturação. Neste trabalho, observamos claramente que o tamanho de grão cresce com o aumento da concentração de cobre no sistema, porém a magnetização decresce. Isto mostra que a característica intrínseca da composição prevaleceu sobre a característica extrínseca, que no caso depende das variáveis de processamento, como é o caso do tamanho de grão. Outro fator que contribuiu para queda da magnetização de saturação foi uma possível mudança nos estados de oxidação de $\mathrm{Cu}^{2+}$ para $\mathrm{Cu}^{+}$. A substituição dos íons $\mathrm{Cu}^{+}$e $\mathrm{Cu}^{2+}$ por níquel nos sítios octaédricos vai resultar em uma queda da magnetização. Este comportamento já foi reportado [16].

Para o caso do campo coercitivo e para as perdas por histerese podemos observar que decrescem com o aumento da concentração de cobre. Isto é justificado pela dependência direta da coercividade com o tamanho do grão, ou seja, maior tamanho de grão menor força coerciva. E como observado na Tabela II, o tamanho de grão é maior para maior concentração de cobre.

No estudo das propriedades magnéticas de pós preparados por síntese de combustão de nanopartículas de ferritas Ni-Zn pura e dopada com samário foi observado que para o sistema puro $\left(\mathrm{Ni}_{0.5} \mathrm{Zn}_{0.5} \mathrm{Fe}_{2} \mathrm{O}_{4}\right)$ o valor da magnetização de saturação sinterizada a $1000^{\circ} \mathrm{C}$ resultou em um valor aproximadamente $69 \mathrm{emu} / \mathrm{g}$ [26]. Comparando este valor com os valores de magnetização de saturação obtidos neste trabalho para as dopagens de $0,1,0,2,0,3$ e 0,4 mol de cobre no sistema $\mathrm{Ni}_{1-\mathrm{x}} \mathrm{Cu}_{\mathrm{x}} \mathrm{Zn}_{0,5} \mathrm{Fe}_{2} \mathrm{O}_{4}$, observamos que houve uma redução da magnetização de saturação, ou seja, para o sistema 0,1 temos $68,79 \mathrm{emu} / \mathrm{g}$, uma redução de $0,3 \%$, enquanto para o sistema
0,4 temos $60,24 \mathrm{emu} / \mathrm{g}$, o que equivale a uma redução de $12,7 \%$ com relação ao mesmo sistema puro $\left(\mathrm{Ni}_{0,5} \mathrm{Zn}_{0,5} \mathrm{Fe}_{2} \mathrm{O}_{4}\right)$. Por outro lado, a adição de cobre ocasionou uma redução no valor do $\mathrm{Hc}$ de $25,9 \%$ quando comparando o sistema $\mathrm{Ni}_{0,4} \mathrm{Cu}_{0,1} \mathrm{Zn}_{0,5} \mathrm{Fe}_{2} \mathrm{O}_{4}$ com o sistema $\mathrm{Ni}_{0,1} \mathrm{Cu}_{0,4} \mathrm{Zn}_{0,5} \mathrm{Fe}_{2} \mathrm{O}_{4} 4$, o que mostrou ser uma queda brusca deste parâmetro. As perdas magnéticas também reduziram com o aumento da concentração do cobre como podemos observar nos dados da Tabela III. Os parâmetros campo coercitivo e perdas por histerese obtidos neste trabalho foram um bom indicativo que o aumento da concentração de cobre favorece a produção de dispositivos magneticamente moles ou de alta permeabilidade.

Comparando também os valores de magnetização de saturação obtidos neste trabalho com os valores para sistemas de ferritas $\mathrm{Ni}_{0,8-\mathrm{x}} \mathrm{Cu}_{\mathrm{x}} \mathrm{Zn}_{0,2} \mathrm{Fe}_{2} \mathrm{O}_{4}$, com $0 \leq \mathrm{x} \leq 0,4$ de cobre preparados pelo método soft chemical [11] e sinterizados a $1000^{\circ} \mathrm{C}$ que foi de $68,6 \mathrm{emu} / \mathrm{g}$ para $0,1 \mathrm{~mol} \mathrm{de}$ $\mathrm{Cu}$ e 66,3 emu/g para 0,4 mol, podemos observar que para o sistema $\mathrm{Ni}_{0,4} \mathrm{Cu}_{0,1} \mathrm{Zn}_{0,5} \mathrm{Fe}_{2} \mathrm{O}_{4}(68,79 \mathrm{emu} / \mathrm{g})$ os valores foram semelhantes, enquanto que para o sistema $\mathrm{Ni}_{0,1} \mathrm{Cu}_{0,4} \mathrm{Zn}_{0,5} \mathrm{Fe}_{2} \mathrm{O}_{4}$ $(60,24 \mathrm{emu} / \mathrm{g})$ observou-se uma pequena redução.

\section{CONCLUSÕES}

Os difratogramas de raios $\mathrm{X}$ mostram a fase cristalina da ferrita $\mathrm{Ni}-\mathrm{Zn}-\mathrm{Cu}$ para todos os sistemas em estudo e traços da fase secundária hematita apenas para os sistemas $\mathrm{Ni}_{0,4} \mathrm{Cu}_{0,1} \mathrm{Zn}_{0,5} \mathrm{Fe}_{2} \mathrm{O}_{4}$ e $\mathrm{Ni}_{0,1} \mathrm{Cu}_{0,4} \mathrm{Zn}_{0,5} \mathrm{Fe}_{2} \mathrm{O}_{4}$. O tamanho de cristalito apresentou tendência de crescimento não linear com o aumento da concentração de cobre. O parâmetro de rede também apresentou este mesmo comportamento em função da substituição de um íon de menor raio iônico por outro de maior raio iônico e devido a mudanças nos estados de oxidação do $\mathrm{Cu}^{2+}$ para $\mathrm{Cu}^{+}$.

Foram observados um aumento na densidade e uma diminuição da porosidade aparente com o aumento da concentração de cobre.

Todos os sistemas estudados apresentaram microestrutura 
heterogênea, com grãos de formato irregular e poros intergranulares; o aumento da concentração de cobre favoreceu a o aumento no tamanho de grão e de poros.

A magnetização de saturação, o campo coercitivo e as perdas por histerese diminuíram de uma forma geral de acordo com o aumento da concentração de cobre.

A dopagem do cobre no sistema de ferrita Ni-Zn mostrouse viável para produção de dispositivos magneticamente moles ou de alta permeabilidade, visto que o aumento da concentração do cobre causou uma redução de $0,3 \%$ da magnetização de saturação para o sistema $\mathrm{Ni}_{0,4} \mathrm{Cu}_{0,1} \mathrm{Zn}_{0,5}$ $\mathrm{Fe}_{2} \mathrm{O}_{4}$, enquanto para o sistema $\mathrm{Ni}_{0,1} \mathrm{Cu}_{0,4} \mathrm{Zn}_{0,5} \mathrm{Fe}_{2} \mathrm{O}_{4}$ houve uma redução de $12,7 \%$, comparando com o sistema $\mathrm{Ni}$ Zn puro. Por outro lado a adição de cobre ocasionou uma redução no valor de $\mathrm{Hc}$ de 25,9\% comparando os sistemas $\mathrm{Ni}_{0,4} \mathrm{Cu}_{0,1} \mathrm{Zn}_{0,5} \mathrm{Fe}_{2} \mathrm{O}_{4}$ com o $\mathrm{Ni}_{0,1} \mathrm{Cu}_{0,4} \mathrm{Zn}_{0,5} \mathrm{Fe}_{2} \mathrm{O}_{4}$ e uma redução das perdas por histerese de 15,3\% comparando os sistemas $\mathrm{Ni}_{0,4} \mathrm{Cu}_{0,1} \mathrm{Zn}_{0,5} \mathrm{Fe}_{2} \mathrm{O}_{4}$ e $\mathrm{Ni}_{0,1} \mathrm{Cu}_{0,4} \mathrm{Zn}_{0,5} \mathrm{Fe}_{2} \mathrm{O}_{4}$ com o sistema ferrita $\mathrm{Ni}-\mathrm{Zn}$ puro.

\section{AGRADECIMENTOS}

Ao CNPq e à Rede de Nanotecnologia Molecular e de Interfaces (RENAMI/CNPq) pelo suporte financeiro.

\section{REFERÊNCIAS}

[1] B. V. Bhise, M. B. Dongare, S. A. Patil, S. R. Sawant, J. Mater. Sci. Lett. 10 (1991) 922-924.

[2] C. Y. Tsay, K. S. Liu, T. F. Lin, I. N. Lin, J. Magn. Magn. Mater. 209 (2000) 189-192.

[3] P. S. Anil Kumar, Mater. Lett. 27 (1996) 293-296.

[4] K. Ishino, Y. Narumiya, Am. Ceram. Soc. Bull. 66, 10 (1987) 1469-1474.

[5] M. Sugimoto, J. Am. Ceram. Soc. 82 (1999) 269-280.

[6] S. M. Rezende, A física de materiais e dispositivos eletrônicos, Ed. Univ. Fed. Pernambuco, Recife, PE (1996) 392-411.

[7] S. I. Pyun, J. T. Baek, Am. Ceram. Soc. Bull. 64, 4 (1985) 602-605.
[8] J. D. Lee. Química Inorgânica, 4ª Ed., Ed. Edgard Blucher Ltda., S. Paulo, SP (1997).

[9] J. E. Huheey, Química Inogânica, Princípios de Estructura e Reactividade, Ed. Harla S.A., S. Paulo, SP (1981).

[10] N. Rezlescu, J. Magn. Magn. Mater. 182 (1998) 199206.

[11] J. J. Shrotri, S. D. Kulkarni, C. E. Deshpande, A. Mitra, S. R. Sainkar, P. S. Anil Kumar, S. K. Date, Mater. Chem. Phys. 59 (1999) 1-5.

[12] P. J. Van der Zaag., V. A. M. Brabers, A. N. Jonson, P. F. Bongers, Phys. Rev. B 51 (1995) 17.

[13] R. W. Cahn, P. Haasen, Eds., Physical Metallurgy, North-Holland: Physics Publ., EUA (1983).

[14] M. Guyoy, A. Globus, J. Phys. Suppl. 38, 4 (1977) $157-$ 158.

[15] A. Goldman, Modern Ferrite Technology, Van Nostrand Reinhold, New York, EUA (1990) 15.

[16] I. Z. Rahman, T. T. Ahmed, J. Magn. Magn. Mater. 290-291(2005)1576-1579.

[17] Z. Yue, J. Zhou, L. Li, X. Wang, Z. Gui, Mater. Sci. Eng. B 86 (2001) 64-69.

[18] F. A. P. Cunha, Diss. Mestrado, Universidade Federal da Paraíba, J. Pessoa, PB (2004).

[19] H. Klug, L. Alexander, X-ray Diffraction Procedures, Wiley, New York, EUA (1962) 491.

[20] D. Louer, DICVOL91 for Windows, Laboratoire de Cristallochimie, Université de Rennes I, Campus de Beaulieu, France (1993).

[21] A. C. F. M. Costa, Tese de Doutorado, DEMa, Universidade Federal de S. Carlos, S. Carlos, SP (2002).

[22] A. C. F. M. Costa, M. R. Morelli, L.Gama, R. H. G. A. Kiminami, Mater. Sci. Forum 498, 9 (2005) 618-623.

[23] A. C. F. M. Costa, A. P. A. Diniz, L. Gama, M. R. Morelli, R. H. G. A. Kiminami, J. Metast. Nanocryst. Mater. 20-21 (2004) 582-587.

[24] A. R. Bueno, L. C. F. Gomes, M. C. S. Nóbrega, Anais $41^{\circ}$ Cong. Bras. Cerâm., S. Paulo, SP (1997) 135-138

[25] A .Znidarsic, M. Drofenik, IEEE Trans. Magn. 32, 3 (1996) 1941-1945.

[26] L. Gama, A. P. A. Diniz, A. C. F. M. Costa, S. M. Rezende, A. Azevedo, D. R. Cornejo, Physica B 384 (2006) 97-99.

(Rec. 23/03/2006, Rev. 04/09/2007, Ac. 27/12/2007) 